\title{
Un modelo simplificado para el análisis de datos de deformación de vigas, obtenidos de pruebas de carga en puentes
}

\author{
A simplified model for the analysis of strain \\ of beams, obtained test load bridges
}

Katherine Vargas-Padilla'

Fecha de recepción: 3 de junio de 2014 Fecha de aceptación: 21 de agosto de 2014

\begin{abstract}
Vargas-Padilla, K. Un modelo simplificado para el análisis de datos de deformación de vigas, obtenidos de pruebas de carga en puentes. Tecnología en Marcha. Edición especial Movilidad Estudiantil 20I4. Pág. 21-30.
\end{abstract}




\section{Palabras clave}

Capacidad de carga viva en puentes; análisis de deformación; factores de distribución de carga; losas en acción no compuesta.

\section{Resumen}

Durante la realización de la primera prueba de carga viva en puentes a nivel nacional que lideró el equipo multidisciplinario del proyecto e-Bridge del Instituto Tecnológico de Costa Rica, el 5 de octubre del 2012 en el Puente sobre el río Purires en la Ruta Nacional $\mathrm{N}^{\circ}{ }^{2}$, se registraron, entre otras variables, gran cantidad de valores de deformación en las alas de las vigas principales de la estructura. En ausencia de un mecanismo que facilitara el análisis de estos datos, esta etapa significó un reto importante para todos.

Como alternativa para la correlación de los resultados experimentales con la condición real del puente, se propuso un modelo simplificado mediante el cual fue posible calcular el momento teórico total externo sobre la estructura con el propósito de compararlo con el momento interno experimental calculado a partir de los datos de la prueba.

La correspondencia entre ambos indicó que la losa no participa de manera conjunta con las vigas principales en la toma y distribución de las cargas temporales aplicadas sobre el puente, condición que propicia una repartición no homogénea de los esfuerzos sobre los elementos, y a la vez desfavorece el desempeño global de la estructura; es decir, que con este método se logró detectar fácilmente cuál era uno de los principales problemas estructurales del puente $y$, de otra forma, hubiese sido una comprobación compleja.

\section{Key words}

Bridge live load test; proof test; strain data analysis; load distribution factors; non composite action decks.

\begin{abstract}
During the first live load test in bridges, performed in Costa Ricaon October 05th, 2012 that was managed by the multidisciplinary research project e-Bridge from the Technological Institute of Costa Rica,on the bridge over Purires River on National Route $\mathrm{N}^{\circ} 2$, lot of strain data obtain from flanges of the main steel beams of the structure,were mainly produced. The lack of an analysis mechanisms in this data meant an important challenge to assume. As a response to this need, a simplified model was proposed for calculating the total theoretical external moment over the structure for being compared with the internal experimental moment obtained from the proof test.From this comparison, it was possible to determinate thatthe deck acts in a noncomposite action,that condition indicates that the deck and main steel beams do not work together for taking and distributing live load applied on the bridge. This situation forces tothe stresses over structurebeconcentrated on just a pair of elements meanwhile others do not receive the effect of loads applied, affecting the bridge global performance, so this method could detect easily one of the main structural problems on the bridge that otherwise require complex methods.
\end{abstract}




\section{Introducción}

El diseño y ejecución de la primera prueba de carga viva en puentes que se realizó en Costa Rica fue liderada por el equipo multidisciplinario del proyecto de investigación e-Bridge 1.0: "Predicción remota de fallas en puentes" del grupo e-Science del Instituto Tecnológico de Costa Rica, el 5 de octubre del 2012. La prueba se realizó en el puente sobre el río Purires, de la Ruta Nacional N. 2 donde, según estudio de tránsito realizado por este equipo, circulan diariamente alrededor de I I 318 vehículos, de los cuales cerca del 30\% son vehículos pesados (Ortiz, G. et al., 20 I3, p.259).

En vista de la importancia que tiene esta ruta para el país, y tomando como referencia esta composición porcentual del tránsito, una evaluación de la capacidad de carga viva en la estructura es de fundamental interés para las autoridades del Estado a quienes les corresponde garantizar que la vía opere correctamente.

De esta prueba se tomaron los insumos necesarios para realizar el análisis correspondiente a la etapa de Confiabilidad Estructural Del Puente y Capacidad de Carga necesario para determinar el Índice de Confiabilidad de la estructura y culminar, así, la metodología de evaluación propuesta por el proyecto de investigación.

En la figura I se ilustran las etapas que componen esta metodología y es posible observar la relación de estas s. De ahí la importancia tanto de la recolección de datos de la prueba de carga como del análisis de los resultados obtenidos en esta fase del proyecto.

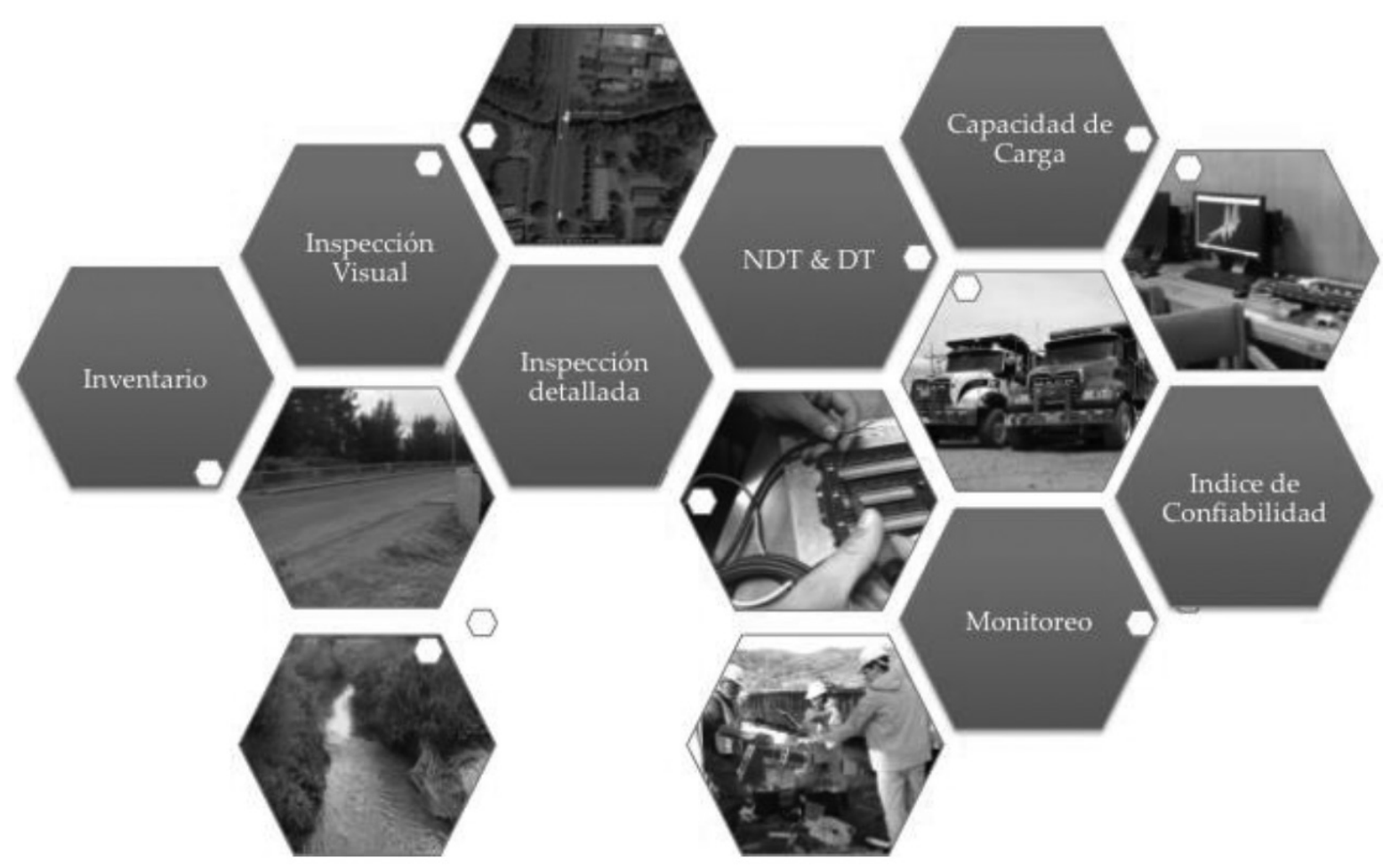

Figura I. Resumen de los elementos utilizados en e-Bridge I.0 para la evaluación de estructuras de puentes. 
Tomando en cuenta que esta fue una experiencia nueva, el mayor reto, una vez culminados los procesos de diseño, planificación y recolección de datos, fue el análisis e interpretación de estos y la correlación con la condición real del puente. En esta fase, fue necesario recurrir a referencias sobre ensayos y pruebas similares desarrolladas en otros países, en donde las pruebas de carga viva en puentes son un parámetro fundamental de comprobación de capacidad, antes de que la estructura sea puesta en funcionamiento.

Desde el punto de vista estructural, el conocer la distribución y condición real de los esfuerzos en los elementos del puente es fundamental para evaluar el comportamiento general de la estructura y, además, un punto de referencia para predecir posibles fallas o anomalías.

Gran parte del trabajo realizado con los datos se enfocó en determinar un método simple de análisis que permita obtener resultados representativos de la condición real del puente ya que uno de los propósitos de este tipo de pruebas es poder identificar los elementos más esforzados para conocer su capacidad de respuesta ante condiciones normales de carga; no obstante, hay una serie de simplificaciones y consideraciones que deben realizarse antes de obtener esa capacidad.

A continuación, se explica en qué consistió el modelo simplificado, la correlación de este con los datos experimentales, y los resultados y conclusiones que derivaron de este.

\section{Marco teórico}

La utilización de sistemas de medición para monitorear la salud estructural en puentes es una práctica común en algunos países de Europa y en los Estados Unidos. La medición de estas variables estructurales y su integración con otras para caracterizar la condición del puente en tiempo real ha sido uno de los avances más importantes. Algunos sistemas consolidados son el Bridge Monitoring System (BRIMOS) que implementa tanto monitoreo permanente con sistemas de medición desarrollados e instalados para proporcionar información sobre el avance de los indicadores clave de desempeño estructural pertinentes en el tiempo; como monitoreo local mediante sensores colocados solamente en algunas posiciones que podrían dar un diagnóstico sobre la situación actual general de la estructura (Vienna Consulting Engineers ZT GmbH, 20 I4) y el AASHTO Ware Bridge Management que además es una herramienta para toma de decisiones en las áreas de preservación, mantenimiento, rehabilitación y/o reemplazo pues ofrecen un modelo de deterioro del puente (AASHTO, 20I4).

Académicamente, se destaca la labor de la Universidad Estatal e Instituto Politécnico de Virginia en Estados Unidos (VirginiaTech), donde este tipo de pruebas se practican con regularidad para evaluar la capacidad de los puentes cuando se acercan a la etapa final de su vida útil. En estas, el desempeño principal y comportamiento característico, usualmente, se enfocan en la deformación de servicio, distribución de la carga, deflexión, efecto dinámico y rotación de los apoyos (Norfleet, W.20 I0, p. 8).

Sin embargo, para el caso de la prueba nacional se generaron datos que corresponden a las dos primeras variables. Eel análisis realizado consistió en obtener los esfuerzos reales y su distribución sobre las vigas principales del tramo central a partir de las deformaciones unitarias medidas en las alas superiores e inferiores. Con estas fue posible calcular el momento flexionante asociado con cada una, bajo condición regular de carga o tránsito normal. Así se logró identificar y cuantificar el efecto de la carga vehicular sobre el puente en estudio, mismo que de otra forma hubiese sido complejo de corroborar sin el uso de instrumentación, dado el desconocimiento de la condición real de los materiales y elementos principales de la estructura.

\section{Metodología}

Para realizar el análisis teórico, se asumieron las siguientes condiciones: el tramo de mayor excitación era el central, la losa y la superestructura actuaban en forma compuesta, los apoyos transferían eficientemente las cargas y la ubicación de estas cargas sobre la estructura era la idónea; no obstante, todas estas fueron simplificaciones para facilitar el modelo que se propuso.

Durante la prueba de carga, los valores de deformación registrados en las alas de las vigas principales, alcanzaron picos de hasta $1000 \mu \varepsilon$; según experiencias de datos registrados por los investigadores de VirginiaTech, en ninguno de los puentes monitoreados por ellos, se había superado 
los $400 \mu \varepsilon$, por lo que estos datos eran extraños y resultó necesario comprobar si estas deformaciones eran o no producto de la carga colocada en ese momento sobre el puente.

Una comprobación simple consistió en determinar si el momento externo producido por el esfuerzo de dos cargas puntuales conocidas solamente sobre una de las vigas de acero del puente (momento teórico) es igual a la suma de los momentos internos en cada viga calculados a partir de las deformaciones unitarias provocadas por las mismas cargas sobre el puente (momento experimental). Si ambos valores concuerdan, entonces, los datos de deformación registrados eran correctos y, por lo tanto, confiables para continuar con el análisis estructural.

Cálculo del momento interno experimental sobre la estructura.

Los sensores con que se midió la deformación en vigas tienen la capacidad de registrar respuestas unitarias provocadas por la dilatación o contracción del material del que está hecho. La deformación está dada por la siguiente ecuación:

$$
\mathrm{Ec}(\mathrm{I}): \varepsilon=\frac{\Delta \mathrm{L}}{\mathrm{L}}
$$

Donde $\Delta \mathrm{L}$ corresponde al cambio en la longitud total (L) de la galga extensiométrica del sensor (Mikhelson, I. and Hicks, T.,20।3, p.5).

Además, el acero de las vigas principales donde se realizó la medición, se debe analizar en su rango elástico pues es aquí donde su falla es dúctil y no ha alcanzado el estado de esfuerzo límite que propicia fallas frágiles y catastróficas para la estructura. Es en este punto donde el valor de su módulo de elasticidad es constante y se puede recurrir a la teoría elástica que establece que la deformación unitaria de un material es directamente proporcional a la fuerza ejercida sobre este, tal como lo expresa la Ley de Hooke:

$$
E c(2): \varepsilon=\frac{\Delta L}{L}=\frac{F}{\left(A^{*} E\right)}
$$

Donde $F$ corresponde a la carga aplicada sobre un área (A) y $E$ corresponde al Módulo de Elasticidad del material (Mikhelson, I. and Hicks, T.,20। 3, p.5).

Una vez determinada esta deformación es posible calcular el esfuerzo mediante la descrita como (Mikhelson, I. and Hicks, T.,20 I3, p.5):

$$
\mathrm{Ec}(3): \delta=\varepsilon^{*} \mathrm{E}
$$

Y obtener el momento flexionante asociado de la siguiente forma:

$$
\operatorname{Ec}(4): M=\frac{(\delta * 1)}{y}
$$

Donde I corresponde al momento de Inercia de la sección y y al módulo de sección relacionado con la posición del eje neutro de los esfuerzos de tensión y compresión en el elemento, variables estrechamente relacionadas con la rigidez (Mikhelson and Hicks, 20।3, p.7).

Este cálculo se realizó para cada una de las cinco vigas principales $y$, luego, se sumaron los resultados para obtener el momento total sobre la estructura.

Modelo simplificado para cálculo de momento externo teórico

Para el cálculo teórico, se tomó solamente una elemento continuo de las mismas propiedades de las vigas principales del puente en estudio, misma cantidad de tramos y condiciones de apoyo, así como distancias entre estos, y se le colocaron dos cargas puntuales "Q y P" que correspondían a las cargas reales por ejes trasero y delantero de las dos camiones utilizados durante la prueba estática en el puente.

Los parámetros de este análisis se muestran en la figura 2 donde se aprecia la magnitud de las cargas aplicadas, las distancias a las que fueron colocadas, las propiedades de la sección utilizada, las condiciones de apoyo del elemento continuo y una imagen del modelo realizado con el programa de análisis estructural SAP2000. 


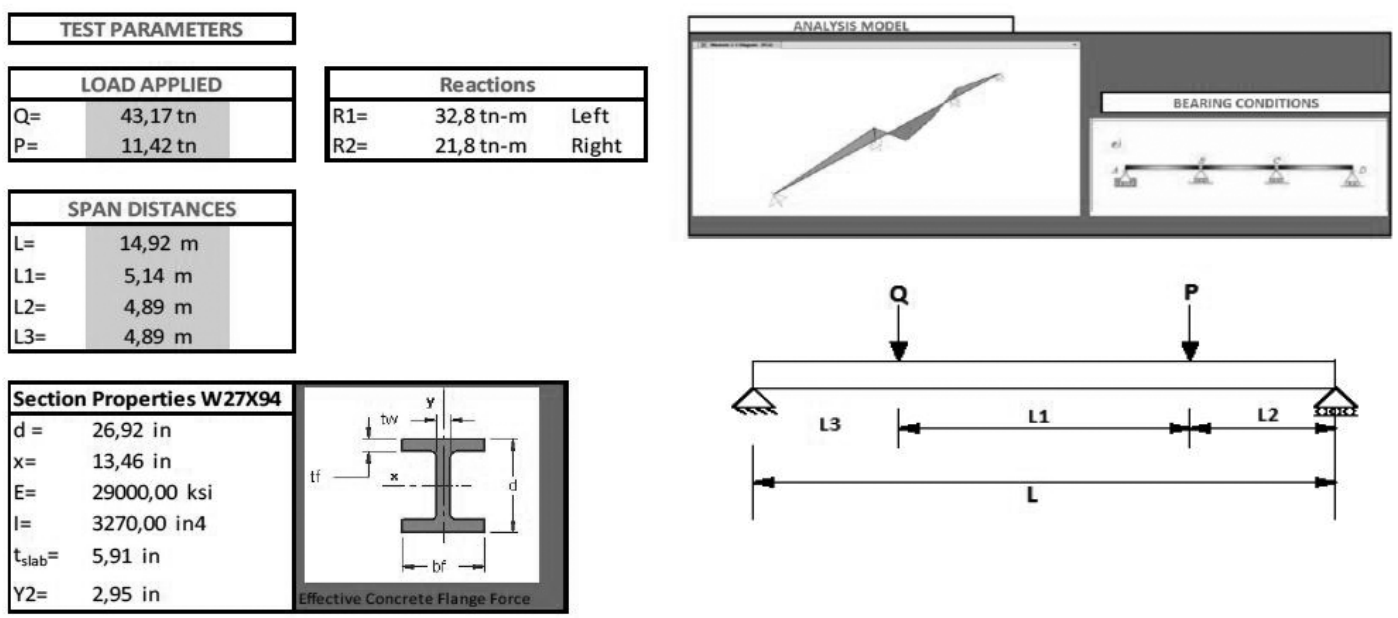

Figura 2. Propiedades de viga de análisis y ubicación de cargas en tramo central para modelo simplificado.

Fuente: Elaboración Propia.

Cálculo teórico de factores de distribución de carga en vigas principales

Los factores de distribución son valores cuantitativos que indican la fracción de carga total del puente que es tomada por cada viga en forma individual (Norfleet, 20 I0, p. 15). Para el caso de la distribución de carga para el puente en estudio, el cálculo teórico de estos factores y su comparación con los experimentales constituyó una herramienta práctica para comprobar la condición real en la estructura. Para esto, se utilizaron como referencia las especificaciones de la American Association of State Highway and Transportation Officials (AASHTO), de 1977. Aunque se desconocía la fecha exacta, se estimó que el puente fue construido alrededor de los años cincuentas por el ejército estadounidense, por lo que la normativa aplicable correspondía a la de esa época.

Estas especificaciones indican que para el caso en que ambos carriles están cargados, la carga que le corresponde a una viga, como elemento estructural primario, está dado por la ecuación:

$$
E c(5): g \_t w o=\frac{S}{\left(5,5 * \frac{S}{(1,676)}\right)}
$$

Donde $S$ es la distancia de máxima separación entre dos vigas paralelas (AASHTO, 1977, p. 56).

\section{Resultados}

De los datos experimentales de deformación, se obtuvo que el momento interno total correspondía a I 804 kip-ft (249942,44kg-m) para la condición de acción compuesta y II 76 kip-ft (I6303।,26 kg-m) en condición no compuesta, tal como se resume en el cuadro I, donde se presentan las deformaciones unitarias tanto en el ala superior (TOP) como en el ala inferior (BOTTON) para cada viga principal ubicadas desde el eje longitudinal B hasta el F. Hubo pérdida de información debido a un daño en el deformímetro colocado en la viga del eje F; para esta se asumió la misma deformación registrada en la viga del eje $B$ pues la estructura era simétrica.

Del modelo simplificado de análisis teórico se obtuvo un momento máximo de 680 kip-ft (94235,03 kg-m) al que le correspondía una deformación de I I $58 \mu \varepsilon$. En la figura 3 se presenta el diagrama de momento flexionante (Moment M3) generado en el SAP 2000 después de realizar el modelo de la viga simple con los parámetros citados anteriormente (Dist Load).

Del cálculo del factor de distribución según la normativa AASTHO 1977 se obtuvo que cada una de las vigas deben tomar alrededor del 30\% del total de carga aplicada.

En el cuadro 2 se presenta el valor de $S$ utilizado y el resultado para las condiciones en que uno o 
Cuadro I. Momento total interno a partir de medición de deformaciones unitarias

\begin{tabular}{|c|c|c|c|c|c|c|c|c|}
\hline \multicolumn{9}{|c|}{ INTERNAL MOMENT } \\
\hline \multirow{2}{*}{$\begin{array}{c}\text { Beam } \\
\text { Strains }(\mu \varepsilon)\end{array}$} & B (TOP) & B (BOTTON) & $\mathrm{c}$ (TOP) & C (BOTTON) & D (TOP) & D (BOTTON) & E (TOP) & E (BOTTON) \\
\hline & 15 & 419 & -273 & 906 & -268 & 1057 & -189 & 445 \\
\hline \multirow{6}{*}{\begin{tabular}{|ll} 
Neutral & $Y 1=$ \\
Axis $x$ & $d-x=c$ \\
& \\
Strains $(\varepsilon)$ & \\
& $\delta=\left(\varepsilon^{*} E\right)$ \\
$I\left(\right.$ in $\left.^{4}\right)=$ \\
Moment $M=\left(\delta^{*} \mid\right) / c$
\end{tabular}} & \multicolumn{2}{|c|}{0.94 in } & \multicolumn{2}{|c|}{6.23 in } & \multicolumn{2}{|c|}{5.45 in } & \multicolumn{2}{|c|}{8.03 in } \\
\hline & \multicolumn{2}{|c|}{25.98 in } & \multicolumn{2}{|c|}{20.69 in } & \multicolumn{2}{|c|}{21.47 in } & \multicolumn{2}{|c|}{18.89 in } \\
\hline & \multicolumn{2}{|c|}{0.000419223} & \multicolumn{2}{|c|}{0.000906213} & \multicolumn{2}{|c|}{0.00105668} & \multicolumn{2}{|c|}{0.000445445} \\
\hline & \multicolumn{2}{|c|}{$12.16 \mathrm{ksi}$} & \multicolumn{2}{|c|}{$26.28 \mathrm{ksi}$} & \multicolumn{2}{|c|}{$30.64 \mathrm{ksi}$} & \multicolumn{2}{|c|}{$12.92 \mathrm{ksi}$} \\
\hline & \multicolumn{2}{|c|}{5640} & \multicolumn{2}{|c|}{4791.93} & \multicolumn{2}{|c|}{4915.7} & \multicolumn{2}{|c|}{4770} \\
\hline & \multicolumn{2}{|c|}{2639 kip-in } & \multicolumn{2}{|c|}{6088 kip-in } & \multicolumn{2}{|c|}{7015 kip-in } & \multicolumn{2}{|c|}{3262 kip-in } \\
\hline & \multicolumn{2}{|c|}{220 kip-ft } & \multicolumn{2}{|c|}{507 kip-ft } & \multicolumn{2}{|c|}{585 kip-ft } & \multicolumn{2}{|c|}{272 kip-ft } \\
\hline$\Sigma$ & \multicolumn{2}{|c|}{1804 kip-ft } & & & \multicolumn{2}{|r|}{$\mathrm{F}$ (assumed) } & \multicolumn{2}{|c|}{220 kip-ft } \\
\hline \multicolumn{9}{|c|}{ Noncomposite Action } \\
\hline & \multicolumn{2}{|c|}{1530 kip-in } & \multirow{2}{*}{\multicolumn{2}{|c|}{$\frac{4154 \text { kip-in }}{346 \text { kip-ft }}$}} & & kip-in & & kip-in \\
\hline & & & & & & kip-ft & & kip-ft \\
\hline$\Sigma$ & & & & & & $\mathrm{F}$ (assumed) & & ip-ft \\
\hline
\end{tabular}

Fuente: Elaboración propia
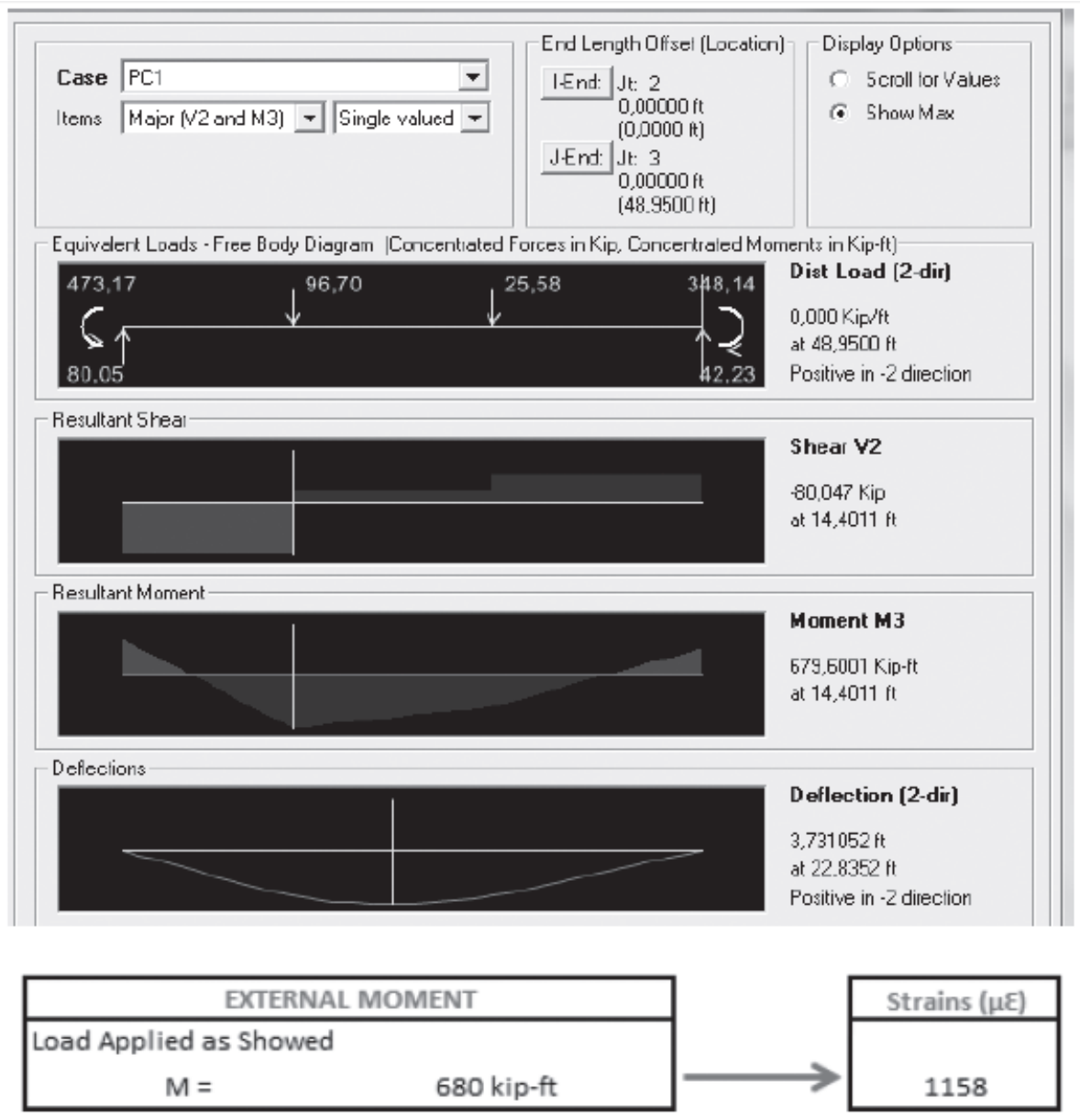

Figura 3. Momento Máximo obtenido de Modelo Simplificado para Análisis Teórico.

Fuente: Elaboración propia. 
dos carriles del puente fueran cargados, aunque para este análisis se utilizó el obtenido para uno porque correspondía con la condición de prueba.

Cuadro 2. Porcentaje de carga para una viga principal

\begin{tabular}{|l|l|l|l|}
\hline \multicolumn{4}{|c|}{ Distribution Factors AASHTO 1977} \\
\hline $\mathrm{S}_{\text {case }}=$ & $172,50 \mathrm{~cm}$ & $5,66 \mathrm{ft}$ & \\
\hline & $\mathrm{g}_{\text {eint one }}=$ & 0,304857 \\
\cline { 2 - 4 } & $\mathrm{g}_{\text {eint two }}=$ & $\mathbf{0 , 3 0 4 7 2 7}$ \\
\hline
\end{tabular}

Fuente: Elaboración propia

Al comparar los valores con la distribución real de carga se evidenció que la viga central es la más cercana a la distribución esperada, mientras que las demás vigas tomaron solamente la tercera parte de lo que teóricamente les correspondía. En el cuadro 3 se muestra el porcentaje de carga que tomó cada viga bajo las dos condiciones analizadas (compuesta y no compuesta) y se comparó, además, el de la viga más esforzada con el valor teórico del AASHTO que se calculó con la ecuación 5, citada anteriormente.

\section{Discusión}

En la etapa de diseño de un puente, los ingenieros asumen ciertas características físicas y mecánicas de los elementos y la forma de funcionamiento e interacción con otros. Algunas premisas importantes son la repartición de cargas y la distribución de esfuerzos sobre la estructura, aspectos relacionados directamente con la rigidez. Aunque parte de estas previsiones se cumplen en esta fase, no es posible conocer con certeza cómo actuará la estructura en condiciones reales de carga, en vista de las variables involucradas durante el proceso constructivo y la dificultad para determinar ciertas características del desempeño. Sin embargo, están deben ser lo más cercanas posible pues, de lo contrario, podría representar anomalías en el comportamiento de la estructura.

\section{Comparación de momentos}

Los datos recolectados durante la prueba indicaron que la viga mayormente esforzada es la ubicada en el eje D, con una deformación de $1057 \mu \varepsilon$ en el ala inferior asociada con un momento máximo de 585 kip-ft (81069,84 kg-m).La variación de las deformaciones y la distribución de los momentos correspondientes a estas se ilustran en la figura 4 donde, además, se indica la posición de la carga con respecto a las vigas principales (ejes B-D).

Ahora bien, al realizar la comparación contra el valor teórico del modelo simplificado, se observó que, bajo acción compuesta, el momento experimental es tres veces mayor y representó el doble para acción no compuesta. Esta relación se ilustra en el cuadro 4 , donde se presenta la relación entre momento experimental y teórico (externo).

Esta diferencia está relacionada con la rigidez de la losa, si esta fuera completamente rígida, sería capaz de distribuir equitativamente las cargas; mientras que, si no lo es, la transmitiría directamente bajo el punto de aplicación de esta(Norfleet, W., 2010, p. 25). Lo anteriormente descrito de debe, en parte, a las simplificaciones del modelo de análisis que consideran materiales, transferencias de cargas y apoyos en condiciones ideales.

Factores de distribución de carga

La teoría de ausencia de acción compuesta se reafirmó al observar el cuadro 3 la Viga D asumió la mayor carga y concentró los mayores esfuerzos. En vista de que la distribución de carga en una estructura se relaciona directamente con la rigidez de la losa, los marcos, diafragmas, apoyos y geometría del puente (Norfleet, W., 20।0, p. 15), no resultó extraño que solamente una de las vigas cercanas contribuyó en

\begin{tabular}{|c|c|c|c|c|c|c|}
\hline \multicolumn{2}{|c|}{ Distribution Factors } & \multirow[b]{2}{*}{ B } & \multirow[b]{2}{*}{ C } & \multirow[b]{2}{*}{ D } & \multirow[b]{2}{*}{$\mathrm{E}$} & \multirow[b]{2}{*}{$\mathrm{F}$} \\
\hline & & & & & & \\
\hline Composite & Experimental (Internal) & 0,122 & 0,281 & 0,324 & 0,151 & 0,122 \\
\hline \multirow[t]{2}{*}{ Noncomposite } & Experimental (Internal) & 0,108 & 0,294 & 0,331 & 0,158 & 0,108 \\
\hline & Theoretical (AASHTO 1977) & & & 0,305 & & \\
\hline
\end{tabular}

Cuadro 3. Porcentaje de carga para una viga principal 

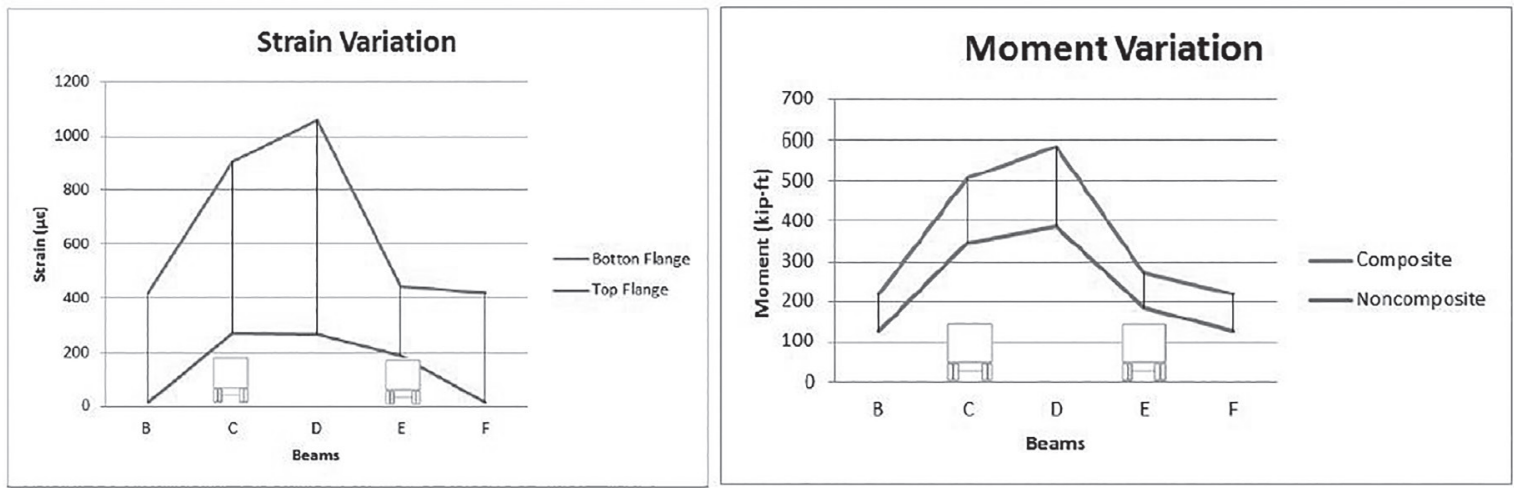

Figura 4. Variación de deformaciones y momentos en vigas principales.

Fuente: Elaboración Propia

poco más del $50 \%$ del valor de carga esperado, y el resto tuviera apenas participación, lo que hizo que esta presentara las mayores deformaciones.

\section{Conclusiones}

A partir del modelo simplificado de análisis teórico se logró determinar, en primera instancia, que deformaciones superiores a los $1000 \mu \varepsilon$ son perfectamente posibles para una estructura de este tipo sometida a las condiciones de carga utilizadas.

Es importante recordar que una prueba de carga puede determinar objetivamente el desempeño estructural actual del puente, mientras que un modelo de análisis lo presenta en forma idealizada; no obstante, la correlación de ambos es fundamental para identificar diferencias en los modos de comportamiento esperados en la estructura.

Para este caso particular, la comparación entre resultados experimentales y teóricos obtenidos del modelo simplificado de análisis, permitió determinar que la condición de transferencia de cargas más representativa es la de acción no compuesta. Esto evidenció que, en la actualidad, la losa no participa activamente en forma integral con los elementos de la superestructura en la toma y repartición de las cargas aplicadas lo que causa una limitación al transferirlas.

Al observar la distribución de esfuerzos, las vigas centrales estaban tomando el mayor porcentaje de carga, mientras que las vigas externas tenían una participación mínima. Se evidenció que la condición actual del puente no favorece una distribución uniforme de los esfuerzos ni deformaciones sobre la estructura y que, por el contrario, se está afectando el desempeño del puente en condiciones de servicio.

Además, es importante resaltar que sin le medición y correlación de estas variables no hubiese sido posible detectar este comportamiento, por lo que se dejó en evidencia la necesidad de realizar pruebas periódicas de carga para poder comparar resultados que permitieran detectar cambios en el desempeño de los puentes en el largo plazo.

Cuadro 4. Relación momento teórico experimental

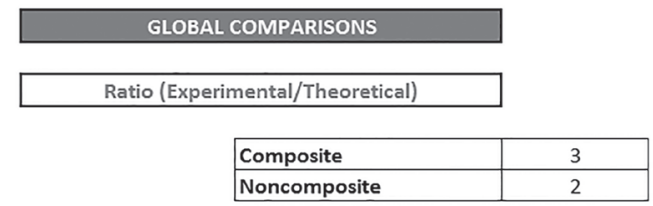

\begin{tabular}{|cc|}
\hline \multicolumn{2}{|c|}{ EXTERNAL MOMENT } \\
\hline Load Applied \\
& $\mathrm{M}=\quad 680 \mathrm{kip}-\mathrm{ft}$ \\
\hline
\end{tabular}

Fuente: Elaboración Propia 


\section{Recomendaciones}

Parte de la experiencia con el análisis de los datos obtenidos en la prueba, evidenció la necesidad de tomar algunas previsiones en el diseño experimental de futuras pruebas, las principales son las siguientes:

\section{a) Prueba previa sensores-Pretest on sensors}

Es importante garantizar la confiabilidad y tener la certeza completa de los instrumentos de medición, antes de realizar la prueba de carga sobre el puente. Es fundamental realizar una prueba de laboratorio en condiciones controladas. Una verificación simple consiste en colocar carga sobre una viga de acero y registrar las deformaciones asociadas; luego, correlacionarlas para obtener los esfuerzos y momentos para, finalmente, compararlos con los resultados de un análisis teórico con ayuda de un programa como SAP 2000, en donde se podría detectar con facilidad si las mediciones corresponden con lo previsto.

b) Medir en todos los tramos-Measurement in all spans

El puente debe instrumentarse en todos los tramos, independientemente del tramo en el que se ubica la carga porque la distribución de esfuerzos y la variación de los momentos debe ser la misma que se observa en el modelo teórico, y si esto no corresponde, se pueden detectar anomalías. Además, esto significa confiabilidad en la medición, de manera que si los momentos registrados son distintos a los previstos, pero su distribución sí corresponde, al menos el parámetro cuestionable será solo la magnitud, y resulta más fácil de detectar el error si, efectivamente, existe.

\section{c) Ubicación de cargas-Ubication of loads}

Durante la prueba, la ubicación de las cargas se definió geométricamente de forma que se colocaron en el centro con base en la premisa de que es el sitio de mayor excitación para la estructura. No obstante, la ubicación de las cargas sobre el puente no se debe definir únicamente a partir de la geometría de las cargas y la estructura sino en parámetros más determinantes como la rigidez, centro de masa y gravedad, tal como lo recomienda el Manual for Steel Construction del AISC, que sugiere algunas reglas generales para colocación de cargas móviles concentradas sobre vigas simples con el objetivo de obtener siempre las máximas respuestas.

\section{Agradecimiento}

Al Ph.D. Roberto León Sáenz, profesor e investigador. Department of Civil and Environmental Engineering. Virginia Polytechnic Institute and State University (VirginiaTech) por su colaboración en la definición del modelo de análisis utilizado y valioso aporte en la interpretación de los resultados obtenidos de dicho modelo. Los autores expresan su agradecimiento al "Programa de Pasantía Estudiantil con Fondos del Sistema 20I4, CONARE-TEC" con el cual se ha cubierto parte fundamental de los gastos de viaje del pasante y a la Rectoría del Instituto Tecnológico que gestiona todo el programa.

\section{Bibliografía}

American Association of State Highway and Transportation Officials Inc.(AASHTO) and Vienna Consulting Engineers ZT GmbH (VCE). (20l4). AASHTOWare. Recuperado desde: http://www.aashtoware.org/Bridge/ Pages/Management.aspx?PID $=2$

American Association of State Highway and Transportation Officials Inc. (AASHTO) Standard Specifications for Highway Bridges. (1977). United States of America: AASHTO.

American Institute for Steel Construction, Inc. (AISC) Manual of Steel Construction Load \&Resistance Factor Design. Volume I. Second Edition. (1994). United States of America: AISC.

BRIMOS BridgeMonitoringSystem. (20 I 4). Hot-Spot Monitoring. Recuperado desde: http://www.brimos.com/ BRIMOS/HTML/de/leistungen/hotspot.html

BRIMOS BridgeMonitoringSystem. (20|4). Permanent Monitoring. Recuperado desde: http://www.brimos.com/ BRIMOS/HTML/de/leistungen/permanent.html

Mikhelson, I. and Hicks,T. (20/3). Structural Engineering Formulas. 2nd Edition. United States of America: McGraw Hill Education.

Norfleet Collins,W. (2010). Live load testing and analysis of the southbound span of U.S. route 15 over interstate 66. "Thesis submitted to the Faculty of the Virginia Polytechnic Institute and State University in partial fulfillment of the requirements for the degree of Master of Science In Civil Engineering", Virginia Polytechnic Institute and State University, Blacksburg, Virginia,USA.

Ortiz, G. et al. (2013). Informe Técnico proyecto e-Bridge I.0: Predicción remota de fallas en Puentes. Cartago, Costa Rica: Instituto Tecnológico de Costa Rica. 